\title{
Preliminary experimental of GPU immersion-cooling
}

\author{
Nugroho Agung Pambudi ${ }^{1}$, Husin Bugis ${ }^{1}$, Ilham Wahyu Kuncoro ${ }^{1}$, Nova Dany Setiawan ${ }^{1}$, Miftah Hijriawan ${ }^{1}$, Bayu \\ Rudiyanto $^{2}$, and Basori Basori ${ }^{1}$ \\ ${ }^{1}$ Mechanical Engineering Education, Univesitas Negeri Sebelas Maret, Jl. Ir. Sutami 36A, Surakarta 57126, Indonesia \\ ${ }^{2}$ Energy Engineering Laboratory, Department of Renewable Engineering, Politeknik Negeri Jember, Jember 68121, Indonesia
}

\begin{abstract}
A typical information technology system takes around $40 \%$ of the total energy used in cooling the system. There are three major classifications of cooling system and they are: water cooling, close loop liquid cooling, and immersion cooling systems. Immersion cooling has been observed to be the latest trend in cooling systems for IT devices. It is a cooling procedure that is carried out through the immersion of all computer components in a dielectric coolant. This research examined the cooling process of GPU using this immersion method. Mineral oil, because of its high dielectric strength, is used as a medium fluid. The temperature difference between the use of fan and immersion cooling was then measured using a benchmark software. The result showed that the immersion cooling produced a lower GPU temperature compared to the conventional fan. The working temperature of the GPU with the use of immersion method was $70^{\circ} \mathrm{C}$ while it was $80^{\circ} \mathrm{C}$ with the conventional fan method.
\end{abstract}

\section{Introduction}

The advancement experienced in information technology has led to the increase in demand for computer and electronic components. According to an estimate carried out by International Telecommunication Union (ITU) in the year 2014, it was discovered that about 3 billion people make use of the internet. This has resulted in a constant strain of environmental and economic resources because of the energy consumed by these systems. This is reflective in a report presented on energy consumption that in the year 2012, computer data centers consumed $270 \mathrm{TWh}$, making up $1.4 \%$ of the total global energy consumption (Leo Grange et al., 2018). It has also been discovered that the next level of energy consumption will be of a tremendous growth compared to the former. By the year 2030, the estimated energy consumption is around $3 \%$ for the best scenario and $13 \%$ for the worstcase scenario. [1].

A typical information technology system would have a mechanism to cool itself and this system takes around $40 \%$ of the total energy used in cooling the system [2]. There are three major classifications of cooling system in computer components and they are: water cooling, close loop liquid cooling, and immersion cooling systems. The process of cooling with a fan is termed as "air cooling". It is a simple conventional method of cooling whereby the heat produced by the electric components are transferred to a conventional fan which blows the heat or inhales it.

This current conventional fan technology is limited in capacity, and because of this, there is need for advanced cooling systems. Furthermore, the continuous decrease in physical size of electronic equipment, the high heat flux generated by the chip and the small space limitations for installing air cooling systems have drawn the attention of a lot of researchers to investigate and improve the efficiency of the heat dissipation processes. An example of this can be seen in the introduction of highperformance liquid cooling systems and mini heat sinks [3-5]. Therefore, the liquid cooling system is employed. This is a process of cooling where the coolant does not come in contact with the components it cools directly. Fluids are pumped and piped through pipes laid adjacent to electrical components. The use of these liquid media will make the movement of heat from electronic components to be more effective [6].

Immersion cooling is the latest trend in cooling systems. It is a cooling procedure that is done through the immersion of computer components in a dielectric coolant [7]. This method works on the principles of convection heat transfer because liquid is the main material used. Immersion cooling technology enables minimization of some common problems encountered while the system is in operation, this is one of its unique benefits. These benefits include reduction in the failure of soldered joints, lowering the working temperature of motherboards, absence of corrosion of electrical contacts, no involvement of moving parts of the equipment, no exposure to electrostatic discharge, and minimal or zero changes in the environment [8]. The technology makes use of different medium coolants (chemical properties) which vary due to the various cooling system used and the electronic equipment on which they are applied. Medium coolants for electronic devices have special requirements: they must be non-flammable, non-toxic, and affordable. It is also important that in addition to 
good chemical and thermophysical properties, the material of the medium must be able to work well with the materials of the cooling components of electronic systems and equipment [9].

Table 1. Heat transfer coefficient on gas and liquid objects

\begin{tabular}{|c|c|c|}
\hline Cooling method & $\begin{array}{c}\text { Heat transfer } \\
\text { coefficient from } \\
\text { air (W/ m2K) }\end{array}$ & $\begin{array}{c}\text { Coefficient of } \\
\text { transfer of heat } \\
\text { from water } \\
\text { (liquid) } \\
\text { (W/ m2K) }\end{array}$ \\
\hline Free Convection & $5-100$ & $100-1200$ \\
\hline $\begin{array}{c}\text { Forced } \\
\text { Convection }\end{array}$ & $10-350$ & $500-3000$ \\
\hline With Steam & - & $3000-100.000$ \\
\hline
\end{tabular}

Immersion cooling is very advantageous because it has a coefficient of transfer of higher interest compared to air as shown in table 1 . In free convection the air heat transfer coefficient is $5-100 \mathrm{~W} / \mathrm{m} 2 \mathrm{~K}$, while in liquid it reaches $100-1200 \mathrm{w} / \mathrm{m} 2 \mathrm{~K}$. That is also the case for the regulated convention, the higher the heat transfer coefficient.

There are general provisions for medium coolant on immersion cooling and can differ depending on the type of cooling system applied and the electronic equipment used. Chemical properties of medium coolants for electronic devices have special requirements, namely: must be non-flammable, non-toxic, and affordable. Medium coolant must have good and qualified thermophysical properties, namely high type heat, high thermal conductivity, and high heat transfer coefficient. The medium is also required in addition to good chemical properties and good thermophysical properties, also in accordance with the material from the cooling system components and electronic equipment (Sohed Murshed and Niel de Castro, 2017).

In this research, preliminary study made use of immersion cooling as the cooling process of GPU (Graphic Processing Unit). Mineral oil was used as the medium fluid because of its high dielectric strength and because it does not contain materials that are harmful to computer devices, humans and the environment.

\section{Method}

\subsection{Graphic Processing Unit}

In this research, we use a Graphics Processing Unit (GPU) off ASUS Redeon RX 550 with specification as shown in Table 2. The schematic diagram is as shown in Fig. 1.

Table 2. RX 550 Spec

\begin{tabular}{|c|c|}
\hline Product Specifications & Radeon RX 550 \\
\hline Compute Units & 8 \\
\hline Stream Processors & 512 \\
\hline
\end{tabular}

\begin{tabular}{|c|c|}
\hline Clock Speeds (Boost / Base) & $1183 \mathrm{Mhz} / 1100 \mathrm{MHz}$ \\
\hline Peak Performance (TFLOPS) & Up to 1.1 \\
\hline Memory Speed (Effective) & Up to $7.0 \mathrm{Gbps}$ \\
\hline Texture Units & 32 \\
\hline ROPs & 16 \\
\hline Memory Bandwidth & $\mathrm{Up}$ to $112 \mathrm{~GB} / \mathrm{s}$ \\
\hline Memory Interface & 128 bit \\
\hline Memory Type & GDDR5 \\
\hline GCN Architecture & $4^{\text {th }}$ Generation \\
\hline AMD FreeSync ${ }^{\mathrm{TM}}$ Technology & Yes \\
\hline $\begin{array}{c}\text { DirectX }{ }^{\circledR} 12 \text { and Vulkan }{ }^{\mathrm{TM}} \\
\text { Support }\end{array}$ & Yes \\
\hline HDMITM & 4K60 support \\
\hline DisplayPort Version & 1.4 \\
\hline HDCP & 2.2 \\
\hline
\end{tabular}

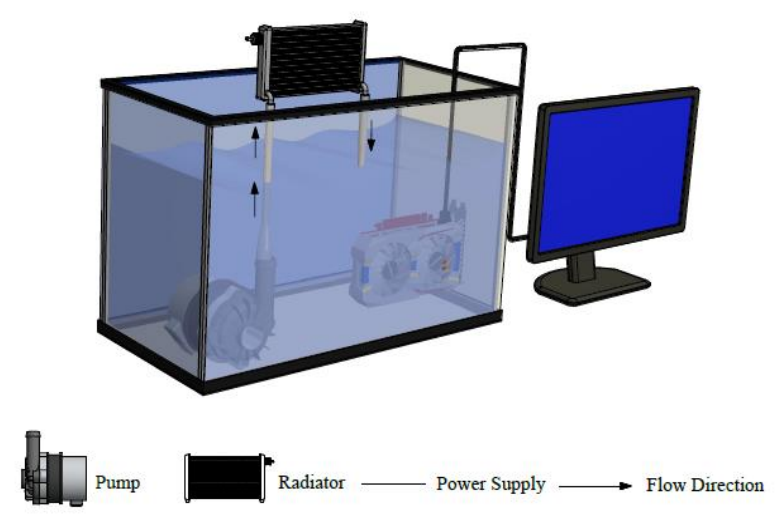

Fig. 1. Schematic diagram.

\subsection{Dielectric Fluid}

Dialectric oil or called mineral oil is composed of paraffin chains of straight, branched and aromatic hydrocarbons with 15 or more carbon (C) in complex mixtures. The physical properties of mineral oil depend on the composition, the composition referred to in the amount of carbon determined by crude oil. Thus, the physical properties possessed by mineral oil variations, ie boiling points range from $300 \mathrm{oC}$ to $600 \mathrm{oC}$, while specific gravity ranges from 0.820 for base oils or light paraffin processes to more than 1.0 for oils in high aromatic processes. Mineral oil is generally insoluble in water and 
alcohol, but can dissolve in chloroform, benzene, ether, carbon disulfide, and protelium ether.

Mineral oil can be applied to engine lubricating oils, automotive and industrial oils, transmission fluids, hydraulic fluids, bearing oils, and coolant oils. Mineral oil in the medical world is used as a medicated oil which consists of a mixture of paraffinic and polythene hydrocarbons obtained by purification. In addition, mineral oil can be used as a laxative and skin care medicine. Mineral oil in commercial applications contains $10-25 \%$ additives. The types of additives contained therein are corrosion inhibitors (inhibitors of corrosion), oxide inhibitors (inhibit oxidation), defoamers (prevent foam formation), and improfer viscosity indexes (reduce the viscosity dependence on temperature) (Aluyor and Ori-Jesu, 2009).

The advantages of using mineral oil for computer cooling systems include not damaging computer components; can provide a simple design compared to conventional cooling using fans; can solve the problem of overheating; and reduce the impact of migration corrosion (Shah et al., 2016a); its dielectric properties do not affect computer reliability, component compatibility; and does not disturb computer systems (Shah et al., 2016b).

In this case, mineral oil was used as the dielectric fluid. According to Eiland et al, the immersion cooling system using mineral oil as the medium has a heat capacity of 1.150 times greater than the use of conventional air cooling as the medium [10]. The oil flowed through a submersible pump connected to a functioning radiator which serves as a cooling device. The heat from the radiator is discharged into the environment. Once cooled, the mineral oil returns to the fluid tub. This cycle is repeated continuously. Total mineral oil placed in the immersion cooling system is 10L (tub, radiator, pipe system). The specification of the mineral oil used is as shown in Table 3 below.

Table 3. Fluid specification

\begin{tabular}{|c|c|}
\hline Mineral oil & \\
\hline Type & No. 15 grade A \\
\hline Kinematic Viscocity & $12.88 \mathrm{~mm}^{2} / \mathrm{s}$ \\
\hline Flash point & $192^{\circ} \mathrm{C}$ \\
\hline Pour point & $-18^{\circ} \mathrm{C}$ \\
\hline Kin. Viscosity Temperatur & $40^{\circ} \mathrm{C}$ \\
\hline
\end{tabular}

\subsection{Experimental Procedure}

Submersible pumps and GPUs are placed in a transparent container, the radiator is installed connected to a pipe connected to a submersible pump. Submersible pumps are used to circulate mineral oil from the container to the radiator, while the radiator releases heat from mineral oil into the air. GPU is connected to a computer device with a screen to monitoring measurement results. The experiment use a benchmark software to find out the maximum performance of the GPU.

\section{Result And Discussion}

\subsection{Temperature Different}

In figure 2 above, there is a graph of time against changes in temperature GPU Thermal Diode. The process in CPU increases due to constant data that is been processes, This data is recorded using temperature logging software.

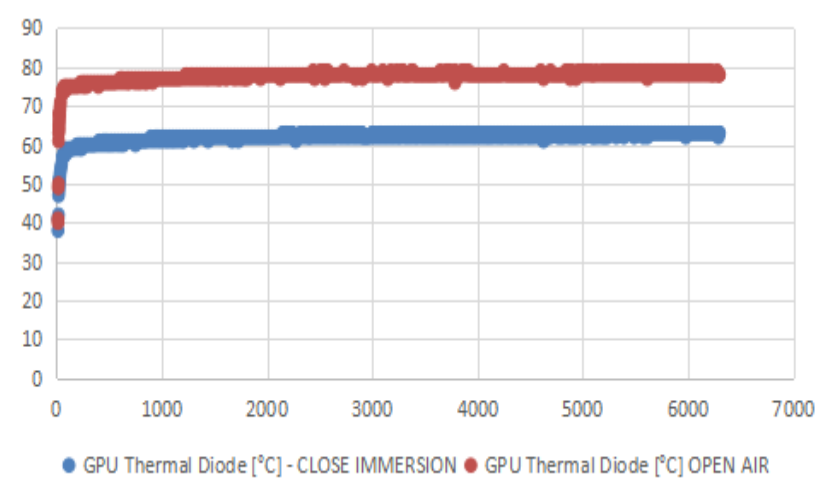

Fig. 2. The temperature difference between fan and immersion cooling experiment.

After three minutes, the GPU reached a stable working temperature and this calls for the comparison of the temperature measurements. Using a cooling fan, GPU reached a temperature of $80^{\circ} \mathrm{C}$ while the use of immersion cooling shows that the temperature measurement of the GPU was $60^{\circ} \mathrm{C}$ with the same benchmark. Form the graph, it can be deduced that there is a difference of up to $20^{\circ} \mathrm{C}$ when immersion cooling method is used compared to the conventional cooling method.

With a decrease in temperature, this will increase the durability of computer components. Work environments with high heat will endanger the components and reduce the working life of the components. this immersion process is very useful. Besides that, when analyzing details about PUE, it will reduce energy consumption. This PUE will be carried out in further research.

\subsection{Visual GPU}

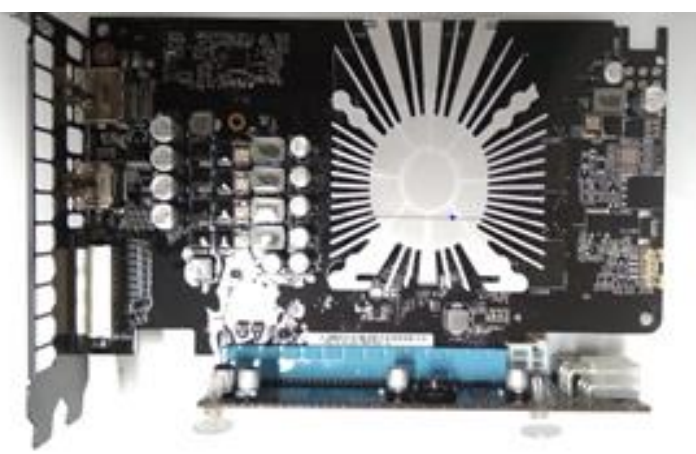

Fig. 3. Fan GPU 


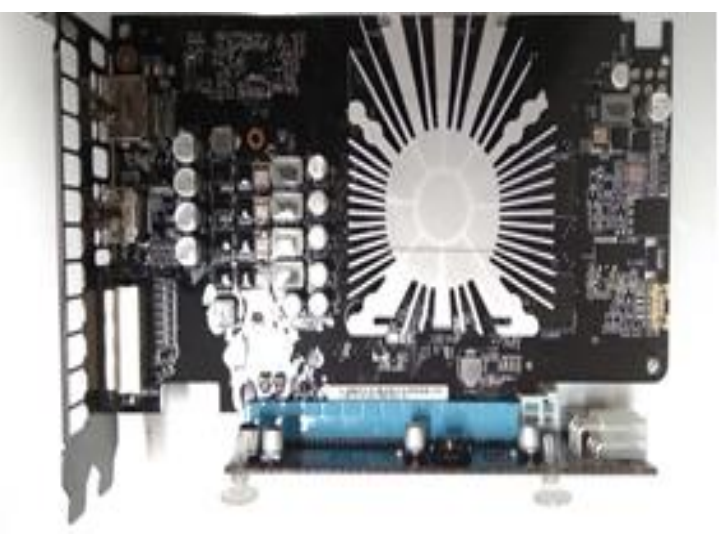

Fig. 4. Immersion GPU

Figures 3 and 4 shows that there is no visual difference between the configuration of fan and immersion cooling method six months after the GPU has been immersed in a mineral fluid.

\section{Conclusion}

This period has witnessed a high rate of advancement in information technology. This development increases the energy consumption used in cooling electronic components, therefore an effective cooling system is needed to reduce this level of energy consumption. Immersion cooling is one of the recent and trending cooling systems. This method uses a dielectric fluid that does not conduct electric current so as to reduce heat effectively.

In the experimental preliminary studies that was carried out, immersion cooling method has been discovered to produce lower GPU temperature when compared with the use of non-immersion cooling systems such as the conventional fan. The result showed that the working temperature of GPU using this method was $70^{\circ} \mathrm{C}$. This is lower than the working temperature of a GPU that uses a fan cooling system.

\section{References}

1. A. Ijam, R. Saidur Nanofluid as a coolant for electronic devices (cooling of electronic devices). Appl. Therm. Eng, 32, 76-82 (2012)

2. Grange, L., Costa, G. D., \& Stolf, P. Green IT for data center powered with renewable energy. Future Generation Computer Systems, 86, 99-120 (2018)

3. Harsh Patel. Immersion Cooling of High End Data Center Server and Validation Through Experiments. University of Texas at Arlington (2013)

4. H.Y. Zhang, D. Pinjala, T.N. Wong, K.C. Toh, Y.K. Joshi. Single-phase liquid cooled microchannel heat sink for electronic packages. Appl. Therm. Eng, 25 1472-1487 (2005)

5. Jimil M. Shah, et al. Reliability Enhancements to Data Center Operation. University of Texas at Arlington (2016).
6. Kheirabadi, A. C. Design of thermal contact liquid cooling system for military server electronics. Dalhousie University (2016).

7. P. Naphon, S. Wiriyasart Liquid cooling in the minirectangular fin heat sink with and without thermoelectric for CPU. Int. Commun. Heat Mass, 36, 166-171 (2009)

8. Richard Eiland, et al. Flow Rate and Inlet Temperature Considerations for Direct Immersion of a Single Server in Mineral Oil. University of Texas in Arlington (2014)

9. Rong, H., Zhang, H., Xiao, S., Li, C., \& Hu, C. Optimizing Energi Consumtion for Data Centers. Renewble and Sustainable Energy Reviews, 58, 674691 (2016)

10. Sohed Murshed S.M. \& Nieto de Castro C.A. A critical review of traditional and emerging techniques and fluids for electronics cooling. Renewable and Sustainable Energy Reviews, 78, 821-833 (2017) 